\title{
Effects of long-term intervention with low- and high-glycaemic-index breakfasts on food intake in children aged 8-11 years
}

\author{
C. Jeya K. Henry ${ }^{1}$, Helen J. Lightowler ${ }^{1}$ and Caroline M. Strik ${ }^{1,2}$ \\ ${ }^{1}$ Nutrition and Food Science Group, School of Life Sciences, Oxford Brookes University, Gipsy Lane Campus, \\ Headington, Oxford OX3 OBP, UK \\ ${ }^{2}$ Human Nutrition Unit, University of Auckland, New Zealand
}

(Received 6 November 2006 - Revised 2 March 2007 - Accepted 2 March 2007)

\begin{abstract}
The aim of the present study was to investigate the effects of long-term intervention of low-glycaemic-index (GI) $v$. high-GI breakfasts on energy and macronutrient intakes in children aged 8-11 years. Preadolescent children were assigned to one of two groups in a random cross-over design. Each group was given low-GI and high-GI breakfasts on two non-consecutive days per week for 10 weeks per breakfast type. Each breakfast provided approximately $1273 \mathrm{~kJ}(300 \mathrm{kcal})$ and was closely matched for macronutrient and dietary fibre content. Subsequent food intake at an ad libitum buffet lunch was recorded and daily energy and macronutrient intakes were measured by $24 \mathrm{~h}$ recall and $3 \mathrm{~d}$ food diaries. There was a tendency towards a reduced energy intake at lunch following the low-GI breakfast compared with the high-GI breakfast, although the mean difference of $75 \mathrm{~kJ}(18 \mathrm{kcal})$ was not significant $(P=0.406)$. In particular, there was a trend towards a reduced energy intake in the lowGI arm compared with the high-GI arm among boys. In addition, data from the $3 \mathrm{~d}$ food diaries showed that there was a tendency towards a reduced energy intake during the low-GI compared with the high-GI study period. In conclusion, although the difference in energy intake following the low-GI and high-GI breakfasts was not statistically significant, the reduced energy intake following the low-GI breakfast is encouraging. Both dietary fibre and carbohydrate type may affect GI, thus their potential and relative modulating effect on appetite requires further investigation.
\end{abstract}

Glycaemic index: Children: Energy intake: Macronutrient intake: Breakfast

Currently there is much interest in the potential health benefits of low-glycaemic index (GI) foods. Epidemiological evidence suggests a beneficial effect of low-GI foods on the incidence of type 2 diabetes ${ }^{1,2}$ and $\mathrm{CVD}^{3}$. A low-GI diet may also have a possible role in the management of obesity through its ability to enhance satiety and modulate appetite ${ }^{4-7}$. Increasing the satiating effect of a food and/or satiety may help curb feelings of hunger that often sabotage weight loss. However, there is debate within the scientific literature, with some studies finding no differences in appetite following low-GI and high-GI foods ${ }^{8-10}$ and some even showing high-GI foods to be more effective ${ }^{11,12}$. To date, there have been few long-term interventions examining the effect of dietary GI on appetite and satiety. The majority of evidence comes from single-day studies, with variable levels of design quality, and most studies have been conducted in adults.

Many previous studies on GI, food intake and satiety have not standardised the amount of dietary fibre, leading to speculation that this may have an additional effect on appetite and satiety, independent of the GI status of the foods. The unique physical and chemical properties of dietary fibre aid in early signals of satiation and enhance or prolong signals of satiety ${ }^{13,14}$. Early signals of satiation may be induced through cephalic and gastric-phase responses related to the bulking properties of dietary fibre on energy density and palatability ${ }^{14}$.
The present study is a follow-on study to recent work $^{7}$, which showed a significant reduction in energy intake at lunch following a low-GI breakfast compared with a highGI breakfast. However, in the Warren study, only a singlemeal effect was investigated. Thus, the present study aimed to examine the effects of a long-term intervention with lowGI $v$. high-GI breakfasts with a similar macronutrient and dietary fibre content on daily energy and macronutrient intakes in preadolescent children.

\begin{abstract}
Materials and methods
Subjects

The primary outcome measure in the present study was the difference in daily energy intake following the low-GI and high-GI breakfasts. Based on a similar study ${ }^{7}$, the within-subject standard deviation of energy intake for three repeated measures was $640 \mathrm{~kJ}(153 \mathrm{kcal})$. Thus, in order to detect a difference in mean daily energy intake of $418 \mathrm{~kJ}$ (100 kcal) with $90 \%$ power at a two-sided significance level of 0.05 , a sample size of twenty-five was required. A target sample of forty was therefore set to allow for dropouts.
\end{abstract}


Thirty-eight children (eleven boys and twenty-seven girls), age 8-11 years, were recruited from a primary school in Oxford, UK. Details about the study were given out at assembly and in the school newsletter. Interested parents and children were provided with a detailed information sheet and consent form to complete and return to the school office. Ethical approval for the study was obtained from the University Research Ethics Committee at Oxford Brookes University (Oxford, UK).

\section{Study design}

A cross-over design was employed with a run-in period (Fig. 1). Subjects were randomly allocated to one of two groups; group 1 or group 2. During the run-in period children were able to sample the breakfasts and on two occasions attended an ad libitum lunch following their usual habitual breakfast at home. Following the run-in period, group 1 consumed a high-GI breakfast for two non-consecutive days each week for a period of 10 weeks. This was followed by a 10-week period when group 2 began a low-GI breakfast. After cross-over, group 1 followed a low-GI breakfast for two non-consecutive days each week for 10 weeks and then group 2 followed a high-GI breakfast for two non-consecutive days each week for 10 weeks. As the two groups did not run simultaneously, this allowed a 'wash-out' period after the high-GI or low-GI breakfast for each group. The study was conducted on two non-consecutive days each week in order to comply with the school's curriculum.

\section{Dietary intervention}

The low-GI and high-GI breakfasts were matched for energy, macronutrient and dietary fibre (dietary fibre as reported by

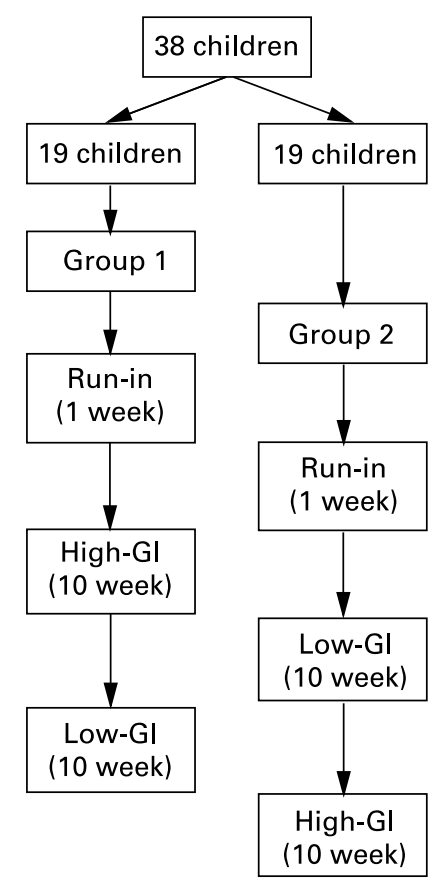

Fig. 1. Study design. Gl, glycaemic index. the food manufacturers) content as far as possible (Table 1). The test breakfasts consisted of:

(1) Low GI: choice of All-Bran (Kellogg's, Warrington, Cheshire, UK), non-Swiss-style muesli (Dorset Cereals Ltd, Dorchester, Dorset, UK), traditional porridge (Quaker Oats, Southall, Middlesex, UK) or soya and linseed bread (Burgen; Allied Bakeries, Maidenhead, Berks, UK) with low-fat spread (Flora, London, UK) and reduced sugar jam (Streamline Foods, Codicote, Herts, UK). All cereals were served with whole milk and all breakfasts were served with $190 \mathrm{ml}$ unsweetened fruit juice.

(2) High GI: choice of Shreddies (Cereal Partners, Welwyn Garden City, Herts, UK), Bran Flakes (Kellogg's), Weetabix (Weetabix, Kettering, Northants, UK) or wholemeal bread (Hovis; British Bakeries Ltd, Windsor, Berks, UK) with low-fat spread (Flora) and jam (Robertson's; RHM Ltd, Marlow, Bucks, UK). All cereals were served with whole milk and all breakfasts were served with $190 \mathrm{ml}$ low-sugar fruit squash (Robinson's, Chelmsford, Essex, UK), with one teaspoon of glucose powder added to increase the GI value of the meals.

The GI values of the breakfast foods were mainly obtained from our own laboratory testing of foods; the few values taken from GI tables ${ }^{15}$ were further confirmed by in vivo testing. The weighted GI of the breakfasts were estimated using the calculation recommended by $\mathrm{FAO} / \mathrm{WHO}:^{16}$

$$
\begin{aligned}
& \mathrm{GI}_{\text {predicted }}= \mathrm{GI}_{(\text {food } \mathrm{a})} \\
& \times \text { carbohydrate }_{(\text {food a })} / \text { available carbohydrate } \\
&+\mathrm{GI}_{(\text {food } \mathrm{b})} \\
& \times \text { carbohydrate }_{(\text {food b) }} / \text { available carbohydrate } \\
&(\text { meal })
\end{aligned}
$$

To quantify the overall glycaemic effect of a given portion of food, the glycaemic load of each breakfast was calculated using the following equation:

$$
\begin{aligned}
& \text { Glycaemic load }=\left(\text { GI }_{\text {breakfast }}\right. \\
& \times \text { weight of available carbohydrate } \text { breakfast }(\mathrm{g})) / 100 .
\end{aligned}
$$

For break-time, children were provided with a small piece of fruit containing approximately $10 \mathrm{~g}$ carbohydrate and were

Table 1. Mean nutritional composition of test breakfasts

\begin{tabular}{lrr}
\hline & Low Gl & High Gl \\
\hline Estimated breakfast Gl & 46 & 77 \\
Breakfast GL & 23 & 43 \\
Energy (kJ) & 1254 & 1252 \\
Energy (kcal) & 300 & 299 \\
Protein (g) & 11 & 8 \\
Carbohydrate (g) & 49 & 56 \\
Fat (g) & 7 & 5 \\
Dietary fibre (g) & 6 & 5 \\
\hline
\end{tabular}

GI, glycaemic index; GL, glycaemic load. 
instructed only to drink water for the period between breakfast and lunch. Lunch was a buffet-style meal and children were allowed free access to a range of foods: a variety of sandwiches (cheese, ham, chicken, tuna, peanut butter, yeast extract), pizza, cherry tomatoes, crisps, cheese sticks, biscuits, cake, fresh fruit, yoghurt, fromage frais, fruit-flavoured beverages and water.

\section{Food intake}

Lunch intake was unobtrusively observed and recorded by trained personnel. A previous study ${ }^{7}$ demonstrated that this method of dietary assessment provided reliable food intake data in children. Daily energy intake was measured by $24 \mathrm{~h}$ recall, conducted by interviewing parents over the telephone. In addition, in a cohort ( $n$ 15), parents completed a $3 \mathrm{~d}$ food diary during each phase of the study, for one study day, one non-study day and one weekend day. Standard household measures and food weights given on packaging were used to estimate food portion sizes in both the $24 \mathrm{~h}$ recall and $3 \mathrm{~d}$ food diary.

Dietary analysis of the lunch intake, $24 \mathrm{~h}$ recall and $3 \mathrm{~d}$ food diary was undertaken using a computerised diet package (WinDiets; Robert Gordon Institute, Aberdeen, UK), supplemented by manufacturers' nutritional and portion size information.

\section{Body mass index}

Height and weight measurements were taken at the baseline and on completion of the study. In addition, body weight was measured at the end of each study cycle. Body weight was measured with an electronic scale to the nearest $0.1 \mathrm{~kg}$ and standing height was recorded to the nearest $\mathrm{cm}$ using a stadiometer (Seca Leicester portable height measure; Seca Ltd, Birmingham, UK), with subjects standing erect and without shoes. BMI was calculated using the standard formula: weight $(\mathrm{kg}) /$ height $(\mathrm{m})^{2}$.

\section{Statistical analysis}

Statistical analysis was performed using SAS/STAT software (version 8; SAS Institute Inc., Cary, NC, USA). Data are presented as means and standard deviations and analysed using a within-subject method. Before statistical analysis, the normality of the data was tested using the ShapiroWilks statistic. Differences in energy and macronutrient intakes at lunch, over $24 \mathrm{~h}$ and over $3 \mathrm{~d}$ between the low-GI and high-GI breakfast study days were compared using the paired $t$ test, or Wilcoxon signed-rank test where appropriate. Statistical significance was set at $P<0 \cdot 05$.

\section{Results}

Of the thirty-eight children recruited, four withdrew and five moved schools during the period of the study. Thus, twentynine children (ten boys and nineteen girls) completed both the low-GI and high-GI arms of the study. Height and weight data at baseline and at the end of the study were obtained from twenty-five children (Table 2). Overall, there was a significant increase in height and body weight due to
Table 2. Body mass index of subjects at baseline and end of study ( $n$ 25) (Mean values and standard deviations)

\begin{tabular}{|c|c|c|c|c|c|c|}
\hline & \multicolumn{2}{|c|}{ Baseline } & \multicolumn{2}{|c|}{ End of study } & \multirow{2}{*}{$\begin{array}{c}\text { Change } \\
(\%)\end{array}$} & \multirow[b]{2}{*}{$P$} \\
\hline & Mean & SD & Mean & SD & & \\
\hline Height (m) & 1.38 & 0.07 & 1.44 & 0.08 & 4 & $<0.001$ \\
\hline Weight (kg) & 33.5 & 5.9 & 38.5 & $7 \cdot 3$ & 15 & $<0.001$ \\
\hline $\mathrm{BMI}\left(\mathrm{kg} / \mathrm{m}^{2}\right)$ & $17 \cdot 7$ & $2 \cdot 4$ & 18.6 & $2 \cdot 9$ & 5 & 0.001 \\
\hline
\end{tabular}

normal growth and development. Mean change in body weight after the low-GI and high-GI study days was 0.6 (SD $0.9) \mathrm{kg}$ and 1.8 (SD 0.6) $\mathrm{kg}$, respectively. The increase in body weight was significantly higher during the high-GI study period compared with the low-GI study period $(P<0 \cdot 001)$. Following the high-GI arm of the study, there was an increase in body weight in all subjects (range $0.7-3.4 \mathrm{~kg}$ ). In comparison, following the low-GI study period, there was an increase in body weight in fifteen subjects (range $0 \cdot 1-2 \cdot 5 \mathrm{~kg}$ ).

Mean energy and macronutrient intakes at lunch following the low-GI and high-GI breakfasts are shown in Table 3. There were no significant differences in intakes between the low-GI and high-GI breakfast study days. The mean difference in lunch energy intake between the low-GI and high-GI breakfast study days was $75 \mathrm{~kJ}$ (18 kcal); however, this was not significant $(P=0.406)$. When the analysis was stratified by sex, there was no significant difference in mean energy intake among boys $(P=0.094)$ or girls $(P=0.372)$, although there was a trend towards a reduced intake in the low-GI arm compared with the high-GI arm among boys (3248 v. $3497 \mathrm{~kJ}$, respectively).

Mean energy and macronutrient intakes over $24 \mathrm{~h}$ for the low-GI and high-GI breakfast study days are shown in Table 4. There was a tendency towards a reduced energy intake over $24 \mathrm{~h}$ on the low-GI breakfast study day compared with the high-GI breakfast study day. However, the mean difference of $254 \mathrm{~kJ}$ (61 kcal) was not significant $(P=0 \cdot 449)$. When the analysis was stratified by sex, there was no significant difference in mean energy intake among boys $(P=0.511)$ or girls $(P=0.594)$, although in both boys and girls there was a trend towards a reduction in food intake following the low-GI compared with the high-GI breakfast $(9022 v .9311 \mathrm{~kJ}$ and 8017 v. $8238 \mathrm{~kJ}$, respectively).

Table 3. Ad libitum energy and macronutrient intake at lunch following test breakfasts

(Mean values and standard deviations)

\begin{tabular}{lrrrrrr}
\hline & \multicolumn{2}{c}{ Low GI } & & \multicolumn{2}{c}{ High GI } & \\
\cline { 2 - 3 } & Mean & SD & & Mean & SD & \\
\hline Energy (kJ) & 3057 & 875 & & 3132 & 829 & 0.406 \\
Energy (kcal) & 731 & 209 & & 749 & 198 & \\
Protein (g) & 17 & 7 & & 19 & 7 & 0.071 \\
Carbohydrate (g) & 96 & 30 & & 95 & 24 & 0.699 \\
Fat (g) & 32 & 9 & & 33 & 10 & 0.142 \\
Dietary fibre (g) & 5 & 2 & & 5 & 2 & 0.218 \\
\hline
\end{tabular}

Gl, glycaemic index. 
Table 4. Energy and macronutrient intakes over $24 \mathrm{~h}$ for the low-glycaemic-index (Gl) and high-Gl breakfast study days

(Mean values and standard deviations)

\begin{tabular}{lrrrrrr}
\hline & \multicolumn{2}{c}{ Low GI } & & \multicolumn{2}{c}{ High GI } & \\
\cline { 2 - 3 } & Mean & SD & & Mean & SD & \\
\hline Energy (kJ) & 8495 & 1550 & & 8749 & 1398 & 0.449 \\
Energy (kcal) & 2030 & 370 & & 2091 & 334 & \\
Protein (g) & 69 & 22 & & 73 & 19 & 0.448 \\
Carbohydrate (g) & 262 & 47 & & 263 & 47 & 0.922 \\
Fat (g) & 77 & 17 & & 84 & 17 & 0.182 \\
Dietary fibre (g) & 15 & 5 & & 16 & 3 & 0.307 \\
\hline
\end{tabular}

Mean energy and macronutrient intakes from the $3 \mathrm{~d}$ food diary for fifteen subjects during the low-GI and high-GI breakfast study periods are shown in Table 5. There was no significant difference in energy intakes between the study day, non-study day or weekend day during the low-GI or high-GI breakfast study periods. Although there were no significant differences in energy intake between the high-GI and low-GI study periods, there was a tendency towards reduced energy intakes during the low-GI compared with the high-GI study period. Similarly, there were no significant differences in macronutrient intakes between the study day, non-study day or weekend day during the low-GI or high-GI breakfast study periods.

\section{Discussion}

To date, there have been few long-term studies examining the effects of GI on food intake and most studies have been conducted in adults. The present study is the first to investigate the effects of long-term intervention with low- and high-GI breakfasts on daily food intake in children. The tendency towards a reduced energy intake at lunchtime and during the rest of the day, as measured by $24 \mathrm{~h}$ recall and $3 \mathrm{~d}$ food diaries, is both consistent and encouraging. Although a difference of $254 \mathrm{~kJ}$ (61 kcal) per d may not in itself seem 'significant', over a period of 1 month this will represent a reduction of $7620 \mathrm{~kJ}(1830 \mathrm{kcal})$. The difference in energy intake over $24 \mathrm{~h}$ and $3 \mathrm{~d}$ suggests that subjects felt fuller for longer after consuming the low-GI breakfast compared with the high-GI breakfast.

Many previous studies on GI, food intake and satiety have not standardised the amount of macronutrients and dietary fibre, leading to speculation that these components may have an additional effect on appetite and satiety, independent of the GI status of the foods. The uniqueness of the present study was to keep the macronutrient and dietary fibre content similar, thus allowing the effect of GI to be investigated.

The present study is a follow-on study to recent research conducted in preadolescent children, which showed that a low-GI breakfast increased satiety and reduced ad libitum energy intake at lunch when compared with a high-GI breakfast $^{7}$. One of the limitations of the Warren study was that the breakfasts were not matched for dietary fibre content, with the low-GI breakfast providing more dietary fibre than the high-GI breakfast. The unique physical and chemical properties of dietary fibre aid in early signals of satiation and enhance or prolong signals of satiety ${ }^{13,14}$. Furthermore the viscosity-producing effects of certain fibres may enhance satiety through intestinal-phase events related to modified gastrointestinal function and subsequent fat absorption ${ }^{13}$. Thus, differences in dietary fibre content may have been partially responsible for the positive findings in the Warren study. In the present study, the dietary fibre content of the breakfasts was closely matched. This may partly explain the similar direction but modest changes in energy intake between the two studies.

Foods with low GI properties do not all exert their effect via the same mechanism. Food properties associated with low glycaemic responses include high contents of soluble fibre ${ }^{17}$, amylose $\operatorname{starch}^{4}$, resistant $\operatorname{starch}^{12}$, fructose ${ }^{18}$, protein or fat as well as intact physical structure of grains ${ }^{19}$ or food $\mathrm{pH}^{20}$. The physiological mechanisms of low-GI foods relate to the various factors, as described above, present in the food. While soluble fibre and $\mathrm{pH}$ may lower GI by slowing gastric emptying $^{21}$, the low GI exhibited by foods with high levels of resistant starch or intact cereal grains results from the reduced availability of starch to digestive enzymes ${ }^{21,22}$. The effects of different fibre and carbohydrate types remain an important area of GI research.

The increase in height, body weight and other anthropometric measurements during the study period was expected due to normal growth and development associated with increasing age. However, it is interesting to note that the increase in body weight was significantly higher during the high-GI study period compared with the low-GI study period. These findings support the growing body of evidence that compared with high-GI diets, low-GI diets have a favourable effect on body weight.

In conclusion, the findings from the present study are in keeping with observations reported previously that food intake is altered following a low-GI compared with a highGI breakfast. Energy intake at lunch following the low-GI

Table 5. Energy and macronutrient intakes over $3 \mathrm{~d}$ during the low-glycaemic-index (GI) and high-Gl breakfast periods in a cohort ( $n$ 15) (Mean values and standard deviations)

\begin{tabular}{|c|c|c|c|c|c|c|c|c|c|c|c|c|c|c|c|c|}
\hline & \multicolumn{4}{|c|}{ Energy intake (kJ) } & \multicolumn{4}{|c|}{ Protein intake $(\mathrm{g})$} & \multicolumn{4}{|c|}{ Carbohydrate intake (g) } & \multicolumn{4}{|c|}{ Fat intake (g) } \\
\hline & \multicolumn{2}{|c|}{ Low Gl } & \multicolumn{2}{|c|}{ High Gl } & \multicolumn{2}{|c|}{ Low Gl } & \multicolumn{2}{|c|}{ High GI } & \multicolumn{2}{|c|}{ Low GI } & \multicolumn{2}{|c|}{ High Gl } & \multicolumn{2}{|c|}{ Low GI } & \multicolumn{2}{|c|}{ High GI } \\
\hline & Mean & SD & Mean & SD & Mean & SD & Mean & SD & Mean & SD & Mean & SD & Mean & SD & Mean & SD \\
\hline Study day & 6889 & 1406 & 7511 & 1778 & 50 & 14 & 51 & 19 & 228 & 58 & 249 & 65 & 63 & 15 & 68 & 19 \\
\hline Non-study day & 6411 & 731 & 6501 & 1652 & 57 & 21 & 46 & 14 & 206 & 38 & 214 & 60 & 58 & 10 & 61 & 26 \\
\hline Weekend day & 6297 & 2307 & 6654 & 2381 & 53 & 20 & 54 & 20 & 201 & 80 & 222 & 99 & 58 & 29 & 59 & 23 \\
\hline
\end{tabular}


breakfast was not statistically significantly lower when compared with the high-GI breakfast. However, the difference in energy intake over $24 \mathrm{~h}$ and $3 \mathrm{~d}$ represented $254 \mathrm{~kJ}$ (61 kcal) and $356 \mathrm{~kJ}$ ( $85 \mathrm{kcal})$, respectively. This would account for a total 'energy deficit' of about $111508 \mathrm{~kJ}$ (26651 kcal) over 1 year. Whilst the difference in energy intake following the low-GI and high-GI breakfasts was smaller than in previous studies, this may be due to the closer matching of dietary fibre content of the test breakfasts. Nevertheless, these results suggest that, at least in children, the provision of a low-GI diet may be a dietary strategy to reduce the risk of overweight and obesity.

\section{Acknowledgements}

The present study was funded by an unconditional grant from The Sugar Bureau (London, UK).

\section{References}

1. Salmeron J, Ascherio A, Rimm EB, Colditz GA, Spiegelman D, Jenkins DJ, Stampfer MJ, Wing AL \& Willett WC (1997) Dietary fiber, glycemic load, and risk of NIDDM in men. Diabetes Care 20, 545-550.

2. Salmeron J, Manson JE, Stampfer MJ, Colditz GA, Wing AL \& Willett WC (1997) Dietary fiber, glycemic load, and risk of noninsulin-dependent diabetes mellitus in women. JAMA 277, $472-477$.

3. Liu S, Willett WC, Stampfer MJ, Hu FB, Franz M, Sampson L, Hennekens CH \& Manson JE (2000) A prospective study of dietary glycemic load, carbohydrate intake, and risk of coronary heart disease in US women. Am J Clin Nutr 71, 1455-1461.

4. van Amelsvoort JMM \& Weststrate JA (1992) Amylose-amylopectin ratio in a meal affects postprandial variables in male volunteers. Am J Clin Nutr 55, 712-718.

5. Holt SHA \& Miller JB (1995) Increased insulin responses to ingested foods are associated with lessened satiety. Appetite 24, 43-54.

6. Ludwig DS, Majzoub JA, Al-Zahrani A, Dallal GE, Blanco I \& Roberts SB (1999) High glycemic index foods, overeating, and obesity. Pediatrics 103, e26.

7. Warren J, Henry J \& Simonite V (2003) Low glycemic index breakfasts and reduced food intake in preadolescent children. Pediatrics 112, e414-e419.
8. Barkeling B, Granfelt Y, Bjorck I \& Rossner S (1995) Effects of carbohydrates in the form of pasta and bread on food intake and satiety in man. Nutr Res 15, 467-476.

9. Stewart SL, Black RM, Wolever TMS \& Anderson GH (1997) The relationship between the glycaemic response to breakfast cereals and subjective appetite and food intake. Nutr Res 17, 1249-1260.

10. Ball SD, Keller KR, Moyer-Mileur LJ, Ding YW, Donaldson D \& Jackson WD (2003) Prolongation of satiety after low versus moderately high glycemic index meals in obese adolescents. Pediatrics 111, 488-494.

11. Hospers JJ, Van Amelsvoort JMM \& Weststrate JA (1994) Amylose-to-amylopectin ratio in pastas affects postprandial glucose and insulin responses and satiety in males. J Food Sci 59, 1144-1149.

12. Raben A, Tagliabue A, Christensen N, Madsen J, Holst J \& Astrup A (1994) Resistant starch: the effect on postprandial glycemia, hormonal response, and satiety. Am J Clin Nutr 60, 544-551.

13. Burton-Freeman B (2000) Dietary fiber and energy regulation. J Nutr 130, Suppl. 2S, 272S-275S.

14. Pereira MA \& Ludwig DS (2001) Dietary fiber and body weight regulation. Pediatr Clin North Am 48, 969-980.

15. Foster-Powell K, Holt SH \& Brand-Miller JC (2002) International table of glycemic index and glycemic load values: 2002. Am J Clin Nutr 76, 5-56.

16. Food and Agriculture Organization and World Health Organization (1998) Carbohydrates in Human Nutrition. Report of a Joint FAO/WHO Expert Consultation. Rome: FAO.

17. Holm J \& Bjorck I (1992) Bioavailability of starch in various wheat-based bread products. Evaluation of metabolic responses in healthy subjects and rate and extent of in vitro starch digestion. Am J Clin Nutr 55, 420-429.

18. Guss JL, Kissileff HR \& Pi-Sunyer FX (1994) Effects of glucose and fructose solutions on food intake and gastric emptying in nonobese women. Am J Physiol 36, R1537-R1544.

19. Holt SHA \& Miller JB (1994) Particle size, satiety and the glycemic response. Eur J Clin Nutr 48, 496-502.

20. Liljeberg HGM \& Bjorck IME (1996) Delayed gastric emptying rate as a potential mechanism for lowered glycemia after eating sourdough bread: studies in humans and rats using test products with added organic acids or an organic salt. Am J Clin Nutr 64, 886-893.

21. Pi-Sunyer FX (2002) Glycemic index and disease. Am J Clin Nutr 76, 290S-298S.

22. Bjorck I \& Elmstahl HL (2003) The glycaemic index: importance of dietary fibre and other food properties. Proc Nutr Soc 62, 201-206. 